\title{
The Wicketkeeper and Injury
}

\author{
Mandeep S Dhillon, Sharad Prabhakar, Nirmal Raj
}

\begin{abstract}
Cricket injury has become a significant issue in recent times with many high profile players getting injured during the game. Injury surveillance programs have recently been started and have documented a high injury rate in fast bowlers. One of the most tasking player positions in cricket is wicketkeeping; despite the best protective wear used by them, the nature of the game would imply a high attrition rate due to injury. We looked at the published literature and our own experience, and found little documented data for this subgroup of players. The types of injury recorded and the problems therein are discussed; it is proposed that future focus should be on short-term analysis of injury profiles so as to improve preventive methods, and longterm evaluation of overuse injury or degenerative changes in the body due to the prolonged abnormal posture maintained by wicketkeepers.
\end{abstract}

Keywords: Wicketkeeper, Cricket injury, Sports injury.

How to cite this article: Dhillon MS, Prabhakar S, Raj N. The Wicketkeeper and Injury. J Postgrad Med Edu Res 2013;47(2): 99-102.

\section{Source of support: Nil}

Conflict of interest: None declared

\section{INTRODUCTION}

Wicketkeeping is one of the most difficult specialty positions in cricket and requires immense skill, stamina and concentration. A wicketkeeper needs to maintain full concentration and is expected to receive the ball every single time it is bowled. The unique playing posture involves repetitive crouching and jumping up, in addition to often having to dive for the ball. The wicketkeeper is the only member of the fielding side permitted to wear gloves and external leg guards; ${ }^{1}$ the wicketkeeper may also wear a helmet with a mesh face guard to help protect from injury (Fig. 1).

Cricket injuries have been documented since the last 40 years, ${ }^{2}$ but injury surveillance and documentation of player specific injury profiles is a recent evolution. Although, few studies on cricket injuries do mention injuries related to wicket keeping, the available published data focused on this group of cricketers is scarce. A PubMed search in February 2013 for the keywords 'wicketkeeper' revealed only one case report on uveal melanoma with spontaneous hyphema, ${ }^{3}$ and that too reported in 1997, when wicketkeepers did not routinely wear helmets. However, even in the modern era, with extensive protective gear, eye injuries remain a major issue today; in 2012, Mark Boucher the celebrated South African wicketkeeper was forced to retire from all forms of cricket due to a scleral laceration when a ball ricocheted off the stumps after being bowled by spinner Imran Tahir. The position is thus not without hazards, and the purpose of this review is to present an overview of this specialist player position, and to evaluate the available data related to injuries in wicketkeeping.

\section{EVOLUTION OF WICKETKEEPING}

The origins of cricket go back many centuries, but recorded history actually starts from the 16th century, although the official history of international Test cricket began in 1877. Players have been documented as wicketkeepers since the early 1800s; the first Australian wicketkeeper was Jack Blackham, who played 32 tests between 1877 and 1894.

Indian cricket started almost at the same time, due to the British influence; the first documented first class cricket match was perhaps in 1864, between Madras and Calcutta. Subsequently, the Bombay Presidency Matches were the first documented games, and the team involved was the Parsees. However, despite the team being documented in detail, especially when it toured England, the name of the wicketkeeper is not clearly mentioned. Khershedji Rustomji Meherhomji and Dattaram Dharmaji Hindlekar were two preindependence wicketkeepers who toured England, but the position was brought into prominence by Syed Kirmani, a flamboyant wicketkeeper and recently by MS Dhoni, India World Cup winning keeper batsman.

The wicketkeeper's major function is to stop deliveries that pass the batsman (in order to prevent runs being scored as 'byes'), but he also catches innumerable balls in a match, which come to him by passing the batsman's bat, by edges of the bat, and by collecting high catches or throws from the fielders. Thus, a wicketkeeper is prone to sustain all the injuries described for the other cricketers as a consequence
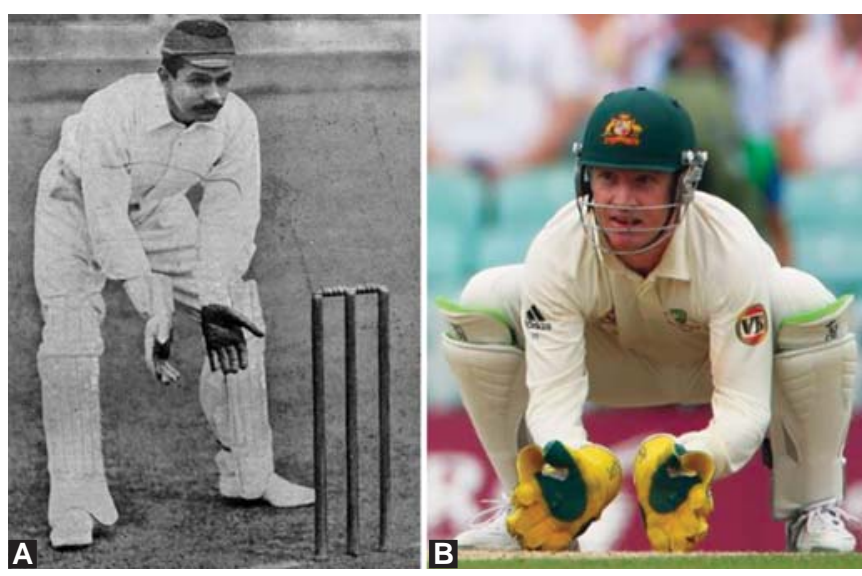

Figs 1A and B: $(A)$ Wicketkeeper protective equipment in the early 20th century; $(B)$ protective equipment today 
of catching, throwing and diving. However, in addition to these injuries, he is exposed to chronic strains of the joints of the back and the lower limb due to the specialized crouching position. With the evolution of modern safety equipment in cricket, there has been a tremendous change in the protective armamentarium available, as compared to the wicketkeepers in the beginning of the 20th century. ${ }^{3}$ In modern day cricket, 'hand safety' has become the principal component of evolution, and this is reflected in the better protective gear of wicketkeepers. A century ago, a wicketkeeper's gloves offered little protection to blows from the ball; ${ }^{2}$ today they have significantly better padding and better webbing between the fingers and thumb (Fig. 2). Protective inner gloves and taping are also worn to considerable benefit. ${ }^{3}$ Additionally helmets with visors, thigh pads and arm guards have come into play, especially when the wicketkeepers of today stand up to even the medium pace bowlers in the shorter form of the game.

\section{INJURY PROFILE OF WICKETKEEPERS}

The nature of the game is such that the wicketkeeper catches the balls while standing still and also while running and diving. It is therefore absolutely essential that a wicketkeeper has an excellent basic catching technique, maintains absolute alertness, and has swift and quick diving skills to give the best performance as well as to avoid injuring

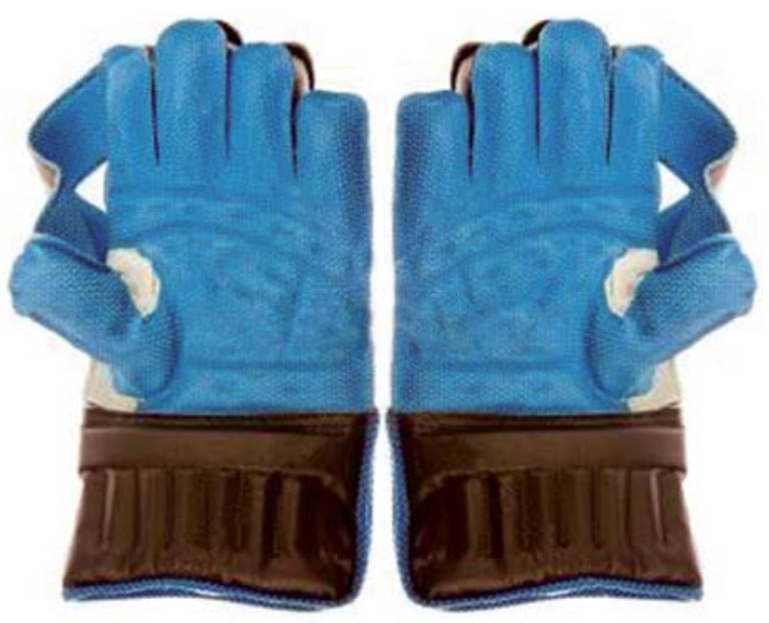

Fig. 2: Wicketkeeper gloves himself. Furthermore, the wicketkeeper is exposed to chronic strains to the back and the lower limb joints due to adoption of the posture of squatting for long spells. Thus, the types of injuries sustained by a wicketkeeper maybe direct hit by the ball, indirect injuries directly related to diving or twisting, overuse injuries, and injuries due to unnatural positions adopted during the game.

Even though a wicketkeeper can sustain a direct injury to any part of his body, his hands are particularly susceptible to injury. In a previous literature review by McGrath and Finch, ${ }^{4}$ most hand injuries in cricket were sustained by wicketkeepers. Examples of indirect injuries are a twisting injury to the knee while turning or throwing a ball or while diving; however, these make up a small percentage of the injuries sustained. Repeated high velocity hits have reportedly lead to osteoarthritis of finger joints and recurrent collateral ligament damage. ${ }^{5}$ There is also some scientific evidence that squatting for prolonged periods may lead to earlier osteoarthritis of the knees; ${ }^{6}$ this is an area of research the present authors are also exploring. Despite the crouching posture, little is published about back problems in wicketkeepers; nevertheless the incidence of stress fractures of the spine in wicketkeepers have been found to be on par with general population. ${ }^{6,7}$

\section{REVIEW OF LITERATURE}

There is no dedicated study looking at injury prevalence among wicketkeepers; even in the injury surveillance studies, the epidemiology and pattern of wicketkeeper's injuries is infrequently documented. McGrath and Finch, ${ }^{4}$ documented the injury types associated with specific cricket player profiles, and found that most hand injuries were sustained by wicketkeepers (Table 1). The authors have reported the injuries to the wicketkeeper as a consequence of direct hit by a bat, hit by a ball, injury received while diving, and injuries caused by collision with another player. ${ }^{4}$

Subsequently Orchard et $\mathrm{al}^{6}$ in 2002, reported a similar incidence of injuries to wicketkeepers; they found $2 \%$ of Australian first class wicketkeepers got injuries of the hand, wrist, lumbar spine, knee cartilage, hamstring strains and knee ligament injuries.

\begin{tabular}{|c|c|c|c|c|c|c|c|}
\hline Cause of injury & Batting & Fielding & Catching & Bowling & Wicketkeeping & Not specified & Total \\
\hline Hit by ball & 20 & 5 & 4 & 3 & 3 & 44 & 79 \\
\hline Hit by bat & - & 1 & 5 & - & 1 & 23 & 30 \\
\hline Fall, slip or trip & 1 & 4 & 3 & - & - & 8 & 16 \\
\hline Overexertion of overuse & - & 1 & 1 & 2 & - & 8 & 12 \\
\hline Finger injury while catching & - & - & 11 & - & - & - & 11 \\
\hline Collision with another player & - & 2 & 1 & - & 1 & 6 & 10 \\
\hline Diving for ball & - & 1 & - & - & - & - & 1 \\
\hline Other & - & - & 3 & - & - & 6 & 9 \\
\hline Total & 21 & 14 & 28 & 5 & 5 & 95 & 168 \\
\hline
\end{tabular}


Many other studies have clubbed the wicketkeeper's injuries with other injuries, thus masking the exact pattern and incidence of these injuries. Stretch, ${ }^{8}$ in a report on South African Cricket Injuries in 2003, has included the injuries of wicketkeepers along with the other fielders, leading to a combined reported rate of $28.6 \%$; injuries were predominantly noted in the upper (40.6\%) and lower (42.9\%) limbs respectively. In another study in 2007, Milson et al have treated the wicketkeeper as a batsman for the purpose of injuries. ${ }^{9}$ Stretch et al in a another recent study documented injuries to wicketkeepers as 47 per 1,000 days. ${ }^{10}$ Looking at chronic cricket related injuries, Corrigan ${ }^{2}$ in 1984 reported that due to repeated squatting the wicketkeepers are prone to osteoarthritis of the knees. However, no subsequent data was collated looking at this important aspect, and this remains a potential area for future research.

In one of the recently published studies on upper injuries in cricketers, Dhillon et al ${ }^{11,12}$ looked at 95 players of the Punjab Cricket Association; this included eight wicketkeepers, and three injuries were seen in these wicketkeepers, with one serious hand injury (Table 2). The overall incidence of injuries to wicketkeepers was noted to be $7 \%$. The number of practice days missed was 55 days, which was second in frequency only to fast bowlers, leading to the conclusion that these specialist players are also injury prone in cricket. The documented injuries to the hand were mainly from direct blows or that sustained while crashing the stumps. The authors also added that wicketkeepers can sustain injuries while batting also, as many of them are good in batting or while throwing the ball.

The authors have not come across any documented head or eye injury in their experience of injury surveillance in

\begin{tabular}{lcc}
\multicolumn{3}{c}{ Table 2: Comparative injury profile of South African and } \\
Indian players
\end{tabular}

local teams over 3 years. However, hand injuries and degeneration was documented, in wicketkeepers and examples are presented below.

Case 1: A 28-year-old State level wicketkeeper sustained an injury to the tip of the little finger. Radiology confirmed an avulsion fracture of the extensor tendon, and a mallet finger was diagnosed (Fig. 3). The patient was advised surgery, which he refused, and he wore a mallet finger splint for 6 weeks, after which he want back to playing over the next 3 months, with a mild extensor lag of the finger.

Case 2: A wicketkeeper who had been playing for his state in the Ranji trophy for 15 years revealed degenerative changes in his finger joints. Despite radiological evidence of arthritis (Fig. 4), his principal problem was stiffness and occasional pain, but this did not stop him from cleanly collecting the ball or throw with the gloves off. His batting skills were also not affected.

The only documented multiple injury in the hands is a press report on David Morrison who played 45 years as an amateur wicketkeeper for the Barton Cricket Club, and has documented fractures of all eight fingers of both hands and both thumbs. ${ }^{13} \mathrm{~A}$ taxi driver by profession, he broke most of his fingers in the 1960s wearing thin leather chamois gloves. He also reported a black eye in 2007 when hit by a ball from a leg spinner.

Many questions are being asked about the excessive tasking of the wicketkeeper during the game, as many feel that the job of wicketkeeper is the toughest role in the field in Test cricket. He/she has to concentrate intensely on every single delivery sometimes for 2 days at a time, and thus the wicketkeepers must be in tip-top condition both physically and mentally. Some are envisaging game protocol changes to minimize unnecessary loads of these players; a unique situation is when every ball played is thrown to the keeper, perhaps due to Cricket Law 23 that states 'the ball becomes dead when it is finally settled in the hands of the wicketkeeper or bowler'. These throws, some with great velocity from fielders standing nearby, may cause unnecessary injury, and many wayward throws lead to the keeper jumping or diving around. This risks preventable injury to the keeper, and could be an avoidable act; the

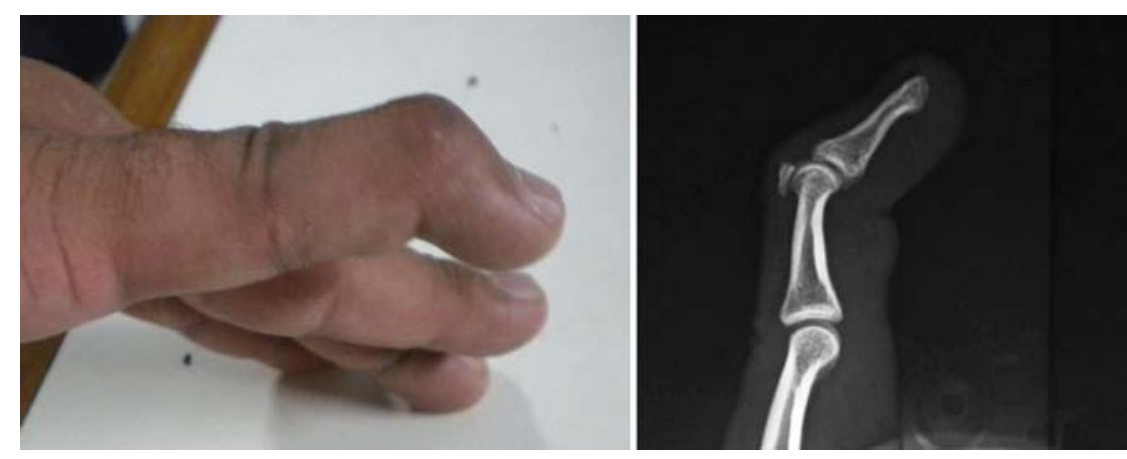

Fig. 3: A 28-year-old wicketkeeper sustained avulsion fracture of extensor tendon of right little finger 

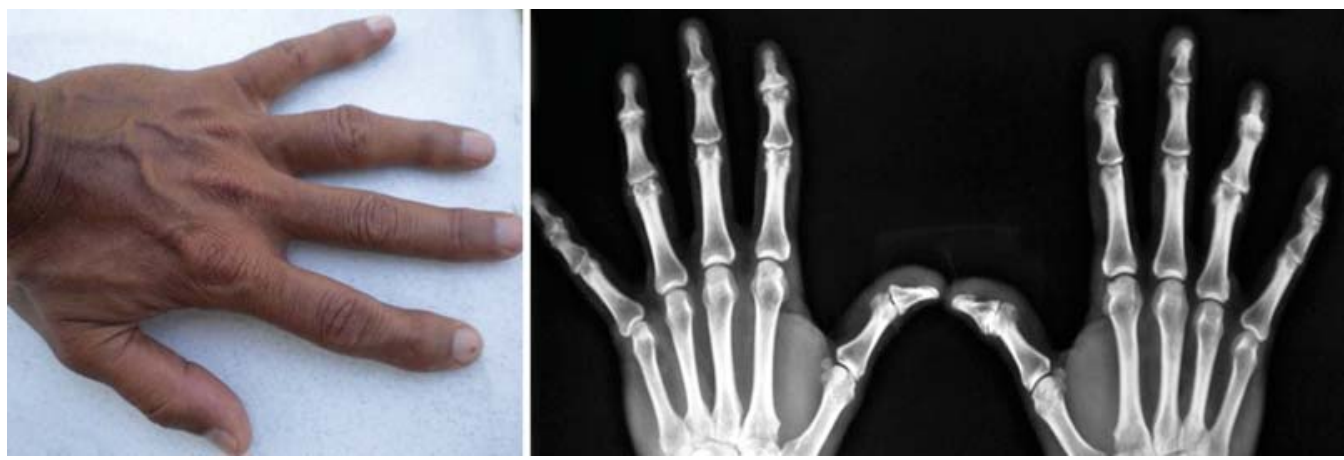

Fig. 4: Degenerative arthritis seen in the PIP and DIP joints of Ranji trophy wicketkeeper playing for last 15 years; he kept on playing without a break

modern fielding teams could be trained to throw the ball toward the bowlers end, or to other fielders shielding the keeper from unnecessary strains.

\section{CONCLUSION}

Although, the wicketkeeper is the only player in the entire cricket team laced with the maximum number of protective equipment including helmet with face shield, pads, special gloves and even mouth guards, they still have the potential for injury as every ball played is directed toward them. Despite the high-risk of injuries, we were not able to find any study in the literature that specifically focused on the pattern, impact and outcome of injuries to wicketkeepers. The available data gives an idea of various possible mechanisms by which a wicketkeeper may be injured; however the studies were mainly pooled up data and are not exclusive for wicketkeepers. A potential area for injury research could be the long-term effects on their hands, as well as the long-term effect on their knees due to bending and lumbar spine due to crouching. A continuous improvement in protective equipment is essential, as even modern day wicketkeepers are getting serious injuries, often due to the changing profile of wicketkeeping, especially when these players stand up close to the stumps. Some changes in playing patterns to shield this specialist player from unnecessary strains when throwing the ball becomes inconsequential, could also be incorporated in the modern sport.

\section{REFERENCES}

1. Law 40 The Wicket Keeper. Lords Home of Cricket. Available from: http://www.lords.org/laws-and-spirit/laws-of-cricket/laws/ law-40-the-wicket-keeper,66,AR.

2. Corrigan AB. Cricket injuries. Australian Family Physician 1984;13(8):558-62.

3. Cher I. Wicket-keeper bowled (over) by (eye) ball. Uveal melanoma with spontaneous hyphema. Med J Aust 1997 Dec 1-15;167(11-12):647.

4. McGrath AC, Finch CF. Bowling cricket injuries over: A review of the literature. Critical Review, 1985-1996. Monash University Accident Research Centre, Wellington Road, Clayton, Victoria,
3168, Australia. Available from: www.monash.edu.au/muarc/ reports/muarc105.pdf.http://www.monash.edu.au/muarc/ reports/muarc105.pdf

5. Belleppa PP, Barton NJ. Hand injuries in Cricketers. J Hand Surg Br 1991 May;16(2): 212-14.

6. Orchard J, James T, Alcott E, et al. Injuries in Australian cricket at first class level. 1995/1996 to 2000/2001. Br J Sports Med 2002;36:270-75.

7. Orchard JW, James T, Portus MR. Injuries to elite male cricketers in Australia over a 10-year period. J Sci Med Sport 2006;9: 459-67.

8. Stretch RA. Cricket injuries: A longitudinal study of the nature of injuries to South African cricketers. Br J Sports Med 2003;37: 250-53.

9. Milson NM, Barnard JG, Stretch RA. Seasonal incidence and nature of cricket injuries among elite South African schoolboy cricketers. SAJSM 2007;19(3):80-84.

10. Stretch RA, Raffan RP, Allan M. Injury patterns of South African provincial cricket players over two seasons. SAJSM 2009;21(4): 151-55.

11. Dhillon MS, Soni RK, Prabhakar S. Epidemiological Study of PCA Cricketers. Presented at 3rd World Congress of Science and Medicine in Cricket, Chandigarh 2012.

12. Dhillon MS, Garg B, Soni RK, Dhillon H, Prabhakar S. Nature and incidence of upper limb injuries in professional cricket players a prospective observation. Sports Med Arthrosc Rehabil Ther Technol 2012 Nov 8;4(1):42.

13. Available from: http://metro.co.uk/2007/09/12/wicket-keeperwith-10-broken-fingers-107805/.

\section{ABOUT THE AUTHORS}

\section{Mandeep S Dhillon (Corresponding Author)}

Professor and Head, Department of Orthopedics, Postgraduate Institute of Medical Education and Research, Chandigarh, India e-mail: drdhillon@gmail.com

\section{Sharad Prabhakar}

Assistant Professor, Department of Orthopedics, Postgraduate Institute of Medical Education and Research, Chandigarh, India

\section{Nirmal Raj}

Assistant Professor, Department of Orthopedics, Postgraduate Institute of Medical Education and Research, Chandigarh, India 